\title{
LOW-COST WHEELED ROBOT-BORNE LASER SCANNING SYSTEM FOR INDOOR AND OUTDOOR 3D MAPPING APPLICATION
}

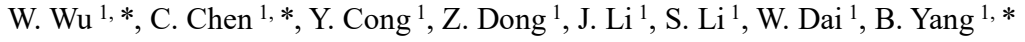 \\ ${ }^{1}$ State Key Laboratory of Information Engineering in Survey, Mapping and Remote Sensing, Wuhan University, No. 129, Luoyu \\ Road, Wuhan, PR China - (weitongwu, chichen, yzcong, dongzhenwhu, lijianping, SenorLee, daiwenxia, bshyang)@whu.edu.cn
}

\author{
Commission II, WG II/3
}

KEY WORDS: Mobile Mapping System, SLAM, Laser Scanning, Low-cost, Robot

\begin{abstract}
:
Aiming to accomplish automatic and real-time three-dimensional mapping in both indoor and outdoor scenes, a low-cost wheeled robot-borne laser scanning system is proposed in this paper. The system includes a laser scanner, an inertial measurement unit, a modified turtlebot 3 two-wheel differential chassis and etc. To achieve a globally consistent map, the system performs global trajectory optimization after detecting the loop closure. Experiments are undertaken in two typical indoor/outdoor scenes that is an underground car park and a road environment in the campus of Wuhan University. The point clouds acquired by the proposed system are quantitatively evaluated by comparing the derived point clouds with the ground truth data collected by a RIEGL VZ 400 laser scanner. The results present an accuracy of $90 \%$ points below 0.1 meter error in the tested scene, showing that its applicability and potential in indoor and mapping applications.
\end{abstract}

\section{INTRODUCTION}

The three-dimensional(3D) mapping of the geometric space is the foundation of various applications such as autonomous driving(Huang et al., 2018), floor plan generation(Liu et al., 2018), construction quality control(Wang et al., 2015) with Building Information Model(BIM), forestry survey(Li et al., 2019), etc. Accurate and real-time ego-motion estimation and environment mapping is the core of these applications.

The traditional Mobile Mapping System(MMS) is equipped with the GNSS/INS system, and the location and orientation of the moving platform are estimated by fusing the GNSS and IMU data directly(Hunter et al., 2006). Currently, most of commercial systems use the integration of GNSS and IMU. However, such MMS is not suit for all kinds of mapping scenes. First, the availability of the GNSS signal. Tall buildings and trees in cities make the GNSS signal unreliable. And, these is no GNSS signal in the indoor scenes. Secondly, the cost of the traditional MMS including high-precision GNSS/INS system is usually more than 300,000 dollars. Thirdly, the mapping results need plenty of post-processing work(Mao et al., 2015; Yu et al., 2015). Therefore, it is necessary to develop a low-cost solution do not rely on the GNSS in indoor and outdoor scenes.

In recent years, the low-cost and multi-sensor approach is a major trend in indoor and outdoor mobile mapping technology(Chen et al., 2017; Jakkola et al., 2010; Li et al., 2019). It is a trend to develop low-cost MMS solutions without the need of positioning or orientation sensors in both indoor and outdoor scenes. Simultaneous localization and mapping(SLAM) is a solution for this demand which can build a 3D map while estimate its current position and orientation of the mobile platform(Smith et al., 1990). In recent decades, two-
dimensional(2D) SLAM algorithms based on the Extended Kalman Filter(EKF) or the particle filter(Grisetti et al., 2007) have achieved great success. However, these algorithms are designed to build the 2D grid map for robot navigation. The 3D SLAM using the multi-beam LiDAR(MBL) remains an active research in recent years(Behley and Stachniss, 2018; Droeschel and Behnke, 2018; Neuhaus et al., 2018; Shan and Englot, 2018). Most LiDAR-SLAM approaches are variations of the traditional scan matching based on iterative closest point(ICP)(Deschaud, 2018).

In this paper, a low-cost two-wheeled robot-borne laser scanning system is proposed, aiming to solve the problem of real-time and accurate 3D laser mapping in GPS denied areas. With the scan to map method, the mapping of surrounding is estimated in realtime, and the global map consistency is achieved by global trajectory optimization after loop closure detection.

The reminder of the paper is organized as follows. In section 2, the system is described in detail. The experiments and analysis of 3D mapping system are presented in Section 3, and conclusions are drawn in the end.

\section{LOW-COST TWO-WHEELED ROBOT-BORNE LASER SCANNING SYSTEM}

\subsection{System Design and Integration}

Our robot-borne laser scanning system (Figure 1) uses the turtlebot3 (ROBOTIS,2017) as the moving platform, which consists of a series of instruments that includes a laser scanner, a IMU and an on-board computer.

\footnotetext{
* Corresponding author
} 


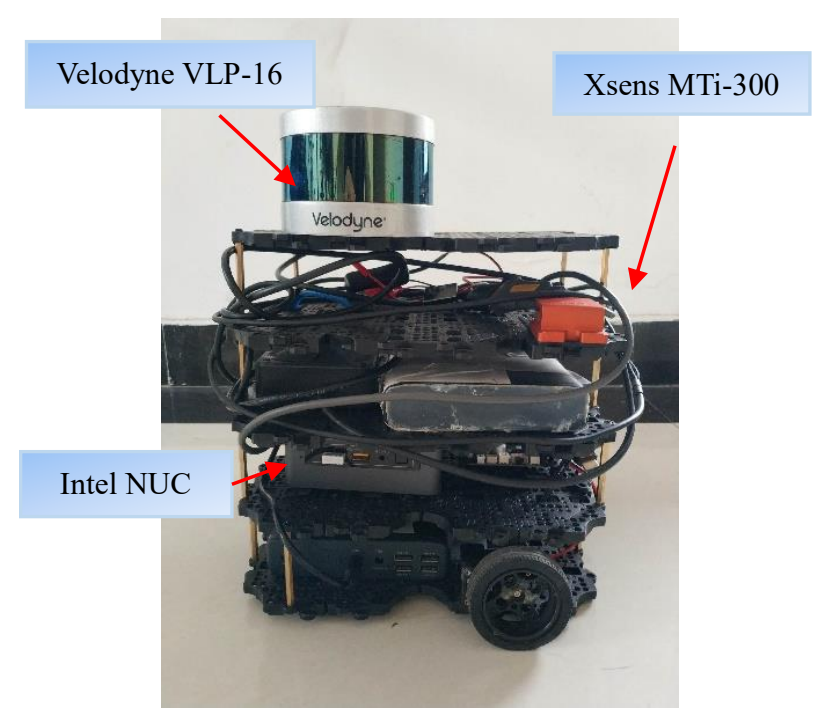

Figure 1. Two-wheeled robot-borne laser scanning system

The laser scanner used in the system is Velodyne VLP-16. It has a 100 meter measuring range and the vertical field of view(FOV) is $\pm 15^{\circ}$. The horizontal angle resolution varies from $0.1^{\circ}$ to $0.4^{\circ}$ based on the rotation rate. In this paper, we use a rotation rate of $10 \mathrm{~Hz}$. The system also equipped with a low-cost inertial measurement unit(IMU), the MTI-300AHRS. The in-run bias stability of the gyroscopes is $10 \% \mathrm{~h}$, and the noise density of the gyroscopes is $0.01 \% \mathrm{~s} / \sqrt{\mathrm{Hz}}$. The in-run bias stability of the accelerometers is $15 \mu \mathrm{g}$, and the noise density of the accelerometers is $60 \mu \mathrm{g} / \sqrt{\mathrm{Hz}}$. The onboard computer is an Intel NUC7i7BNH with an Intel Core i7 processor. Table 1 is the hardware information.

\begin{tabular}{|l|c|}
\hline Equipment & Description \\
\hline Laser scanner & Velodyne VLP-16 \\
IMU & Xsens MTi-300AHRS \\
Onboard computer & Intel NUC7i7BNH \\
\hline
\end{tabular}

Table 1. Hardware Information

The synchronization of the laser scanner and the IMU is done electronically. All data are produced with timestamp. The axes of the MTI-300 are aligned with the VLP-16. The geometry calibration of the VLP-16 and MTI-300 is done mechanically to offer the transform matrix between the adjacent sensor coordinate frames.

\subsection{D Point Cloud Generation}

3D mapping remains an active research in recent years. Many researchers do efforts to achieve real-time six degrees of freedom(6-DOF) SLAM with vision-based and LiDAR-based methods. A low-drift and real-time LIDAR odometry and mapping method is proposed by JiZhang in 2014, named LOAM(Zhang and Singh, 2014). This method novelly divides the estimation problem into two individual algorithms. One algorithm performs odometry at a high frequency and another algorithm performs mapping at a low frequency. To address the problem of mismatching between scattered edge points, LeGOLOAM is proposed(Shan and Englot, 2018). In our system, we adopt LeGO-LOAM as our front end of the proposed SLAM algorithm to generate the point clouds in real time.

The registration error between the current scan to the global map accumulates over time. Therefore, when the mapping system returns to a place which has been visited before, there will be an obvious gap. To achieve a globally consistent map, the system performs global trajectory optimization and get the corresponding mapping point cloud based on a factor graph(Carlone et al., 2014).

\section{EXPERIMENTS AND ANALYSIS}

\subsection{Laser Scanning System's Mapping Result}

In order to verify the accuracy and effectiveness of the proposed system, we experiment the system in both indoor and outdoor scenes respectively. Selected indoor scene is an underground parking $\operatorname{lot}\left(1200 \mathrm{~m}^{2}\right)$. Figure 2 was captured when we use the proposed system to acquire data in the underground parking lot. Outdoor scene is selected in a road environment in Wuhan University. The GNNS signal in the selected outdoor environment is weak, because of the occlusion caused by the trees on the sides of the road, which can be seen from the Figure 3.

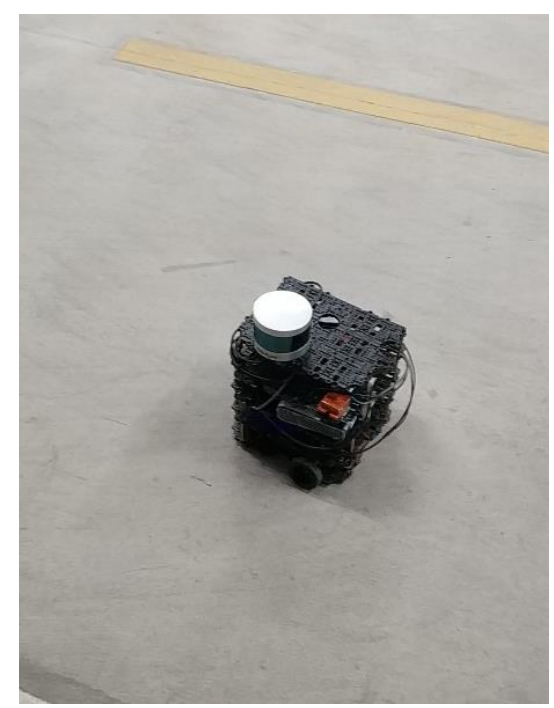

Figure 2. Data collection in underground parking lot

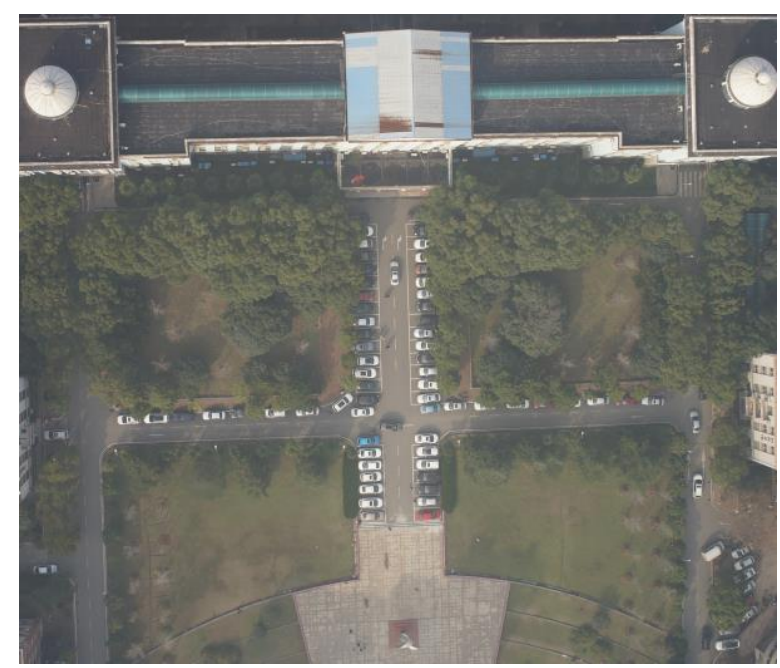

Figure 3.UAV image of the outdoor experimental scene

We use the LiDAR point clouds collected by RIEGL VZ400 TLS laser scanner as the ground truth. Three single scans are registered manually to form the complete parking lot. The point 
clouds collected by VZ-400 and the proposed system are shown in Figure 4. It can be seen from Figure 4 (b) that point clouds collected by our system is of good quality and the geometry of the indoor scene is well preserved.

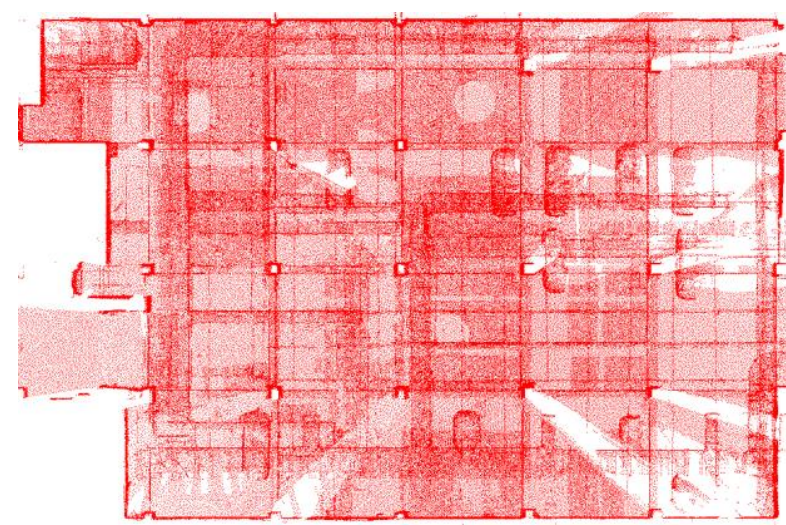

(a) Point clouds of parking lot collected by TLS

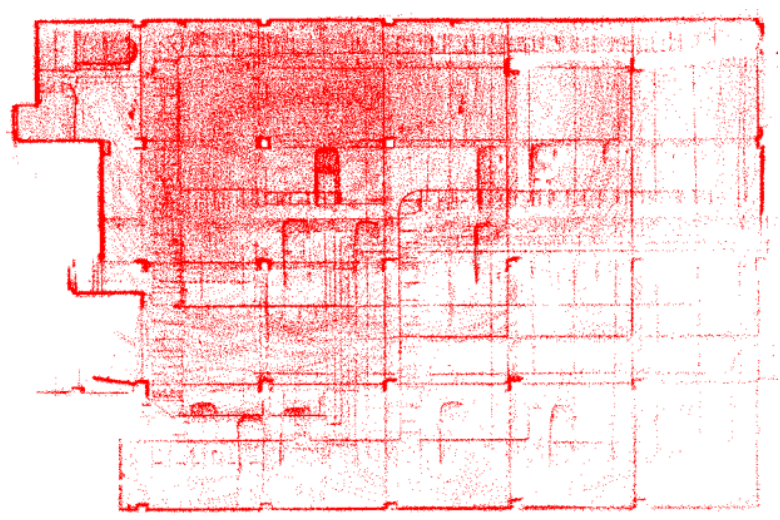

(b) Point clouds of parking lot collected by the robot system

Figure 4. Indoor mapping result in a parking lot

The point clouds collected by the proposed system and the TLS VZ400 in the road environment are shown in Figure 5. From the Figure 5, we can see that the scene includes the road, cars, buildings and trees. From the Figure 6, it reveals that tree trunks and cars have a good mapping result. The shown details verify the system's effectiveness qualitatively.

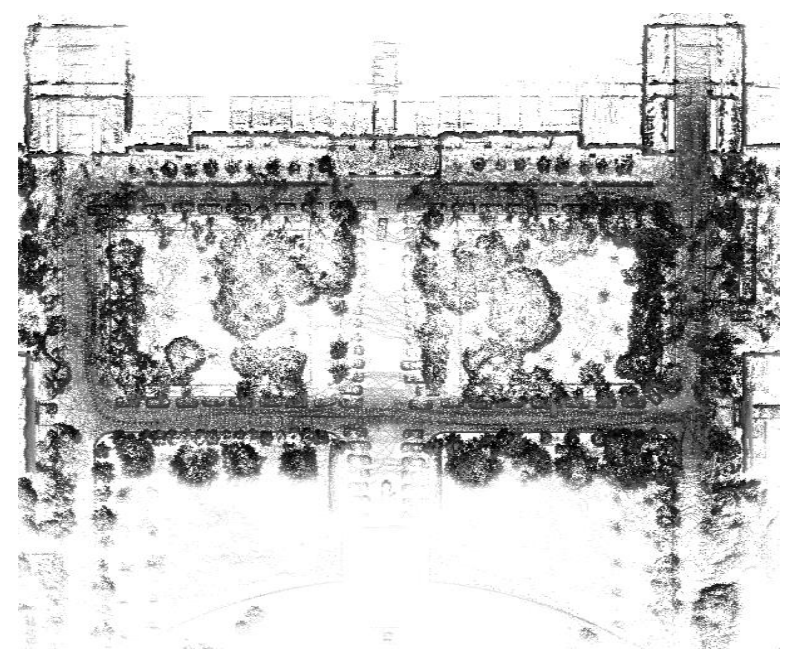

(a) Point clouds collected by the robot system

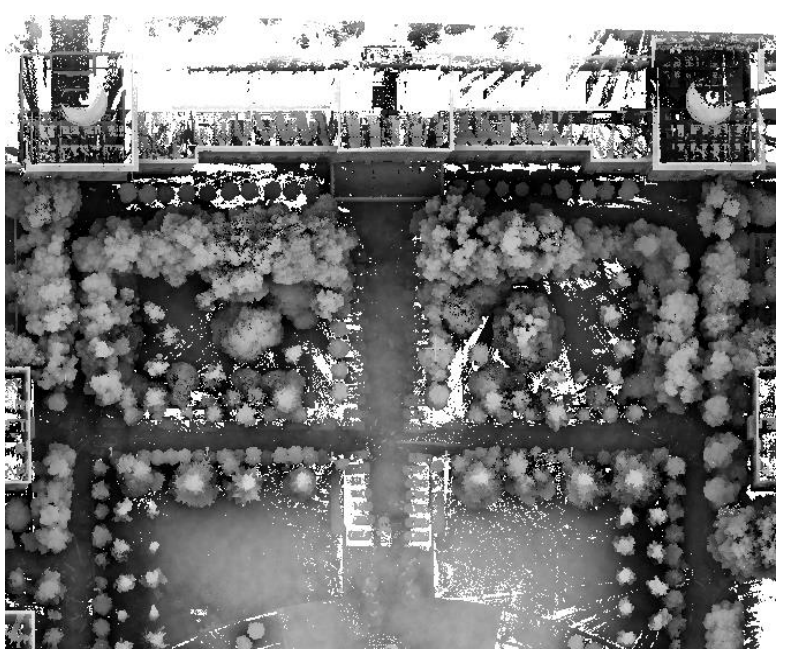

(b) Point clouds collected by TLS

Figure 5. Outdoor mapping result in a road environment

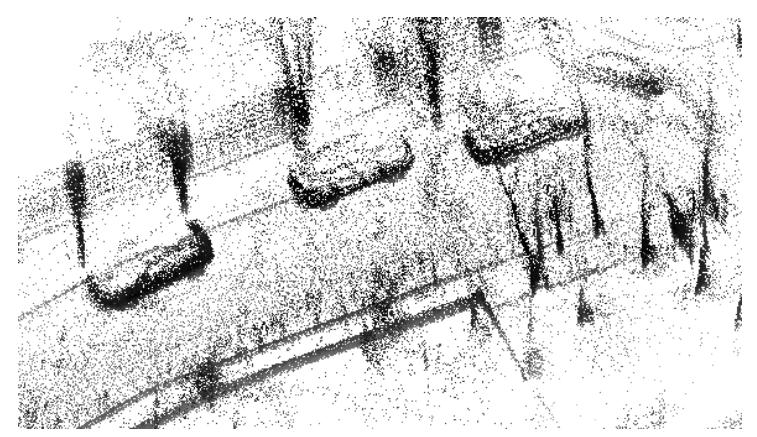

Figure 6. Details in outdoor mapping

\subsection{Robot-Borne Laser Scanning System's Mapping Result Compared with TLS}

To evaluate the accuracy of the point clouds collected by the robot system, we use CloudCompare to calculate the difference against the reference data collected by VZ-400 after these two point clouds are manually registered. The result of distances between two point clouds in the indoor scene is given by Table 2. Overall, the error of $90 \%$ points is within $0.1 \mathrm{~m}$. From the Figure 7 (b), it can be seen that almost all fixed structures such as wall and column are in blue color which means a small difference. Some cars are in yellow or red color because these cars don't exist in the reference data. Since the reference data and the robot mapping data are collected in different time.

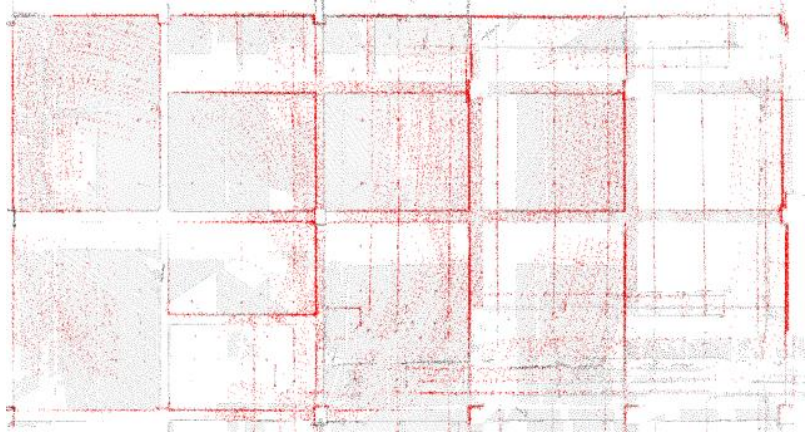

(a) Comparison of the partial upper part of the two point clouds (red represents the system's, black represents the TLS's) 


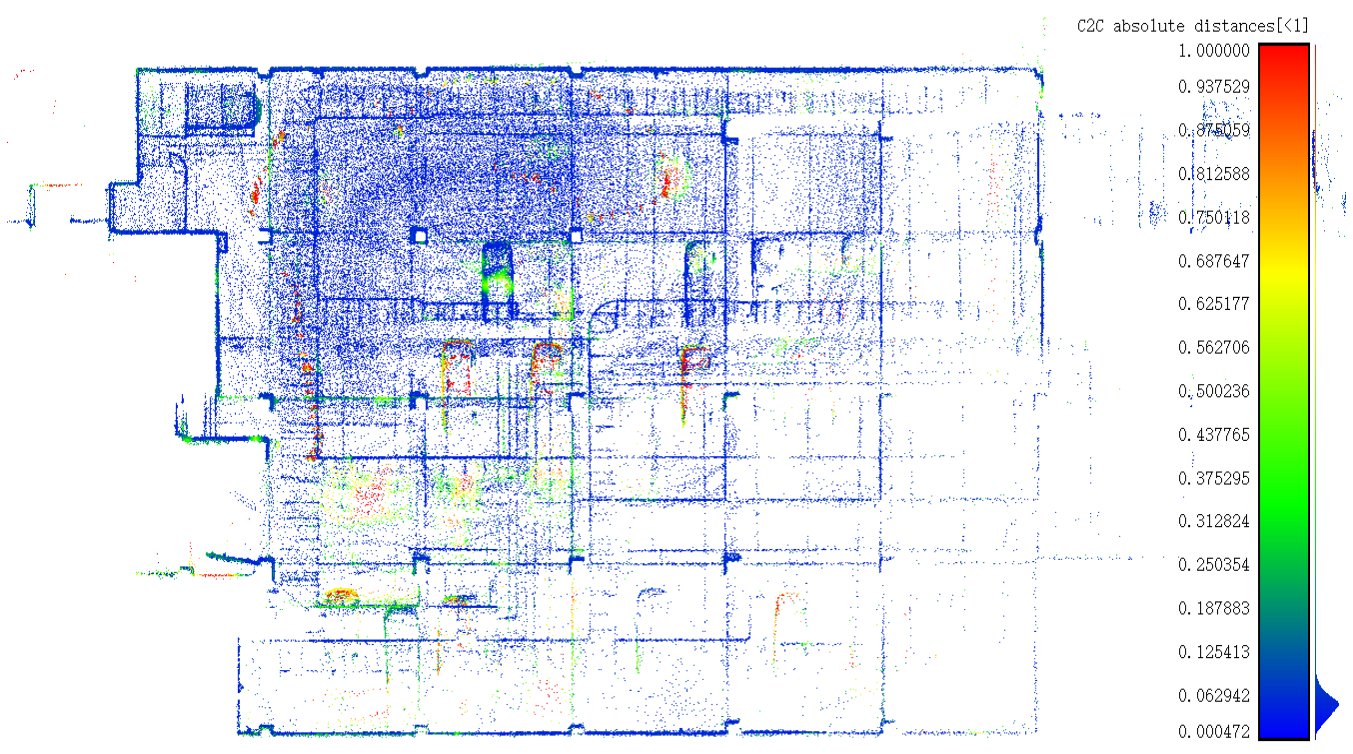

(b) Comparison result of robot system \& VZ400 point clouds with point-to-plane distance

Figure 7. Comparison result of robot system \& VZ400 point clouds in the indoor scene

\begin{tabular}{|c|c|c|c|c|}
\hline Distance(meter) & $0 \sim 0.1$ & $0.1 \sim 0.2$ & $0.2 \sim 0.5$ & $0.5 \sim 1.0$ \\
\hline Percentage(\%) & 90.4 & 3.5 & 3.52 & 1.98 \\
\hline
\end{tabular}

Table 2. Distribution of distance between two point clouds

Given the collecting time of robot-borne system and TLS is different in the campus where the cars and trees differ a lot over time, we only registration two datasets to evaluate the results qualitatively. The registration results are shown in Figure 8 (a). As it can be seen from the Figure 8 (b), the columns collected by our system have a good geometric shape and match the highprecision point clouds highly.

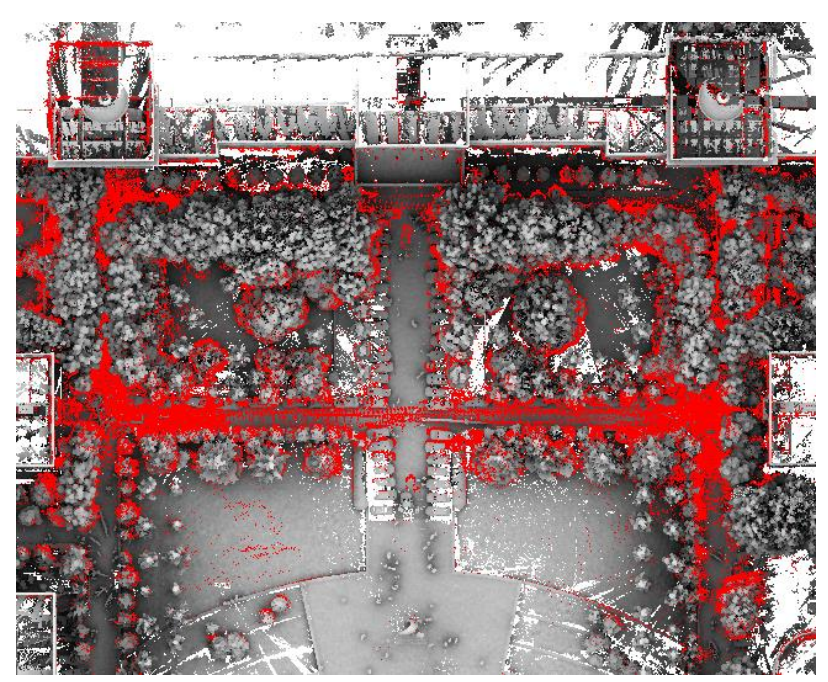

(a) Overlay display of two point clouds

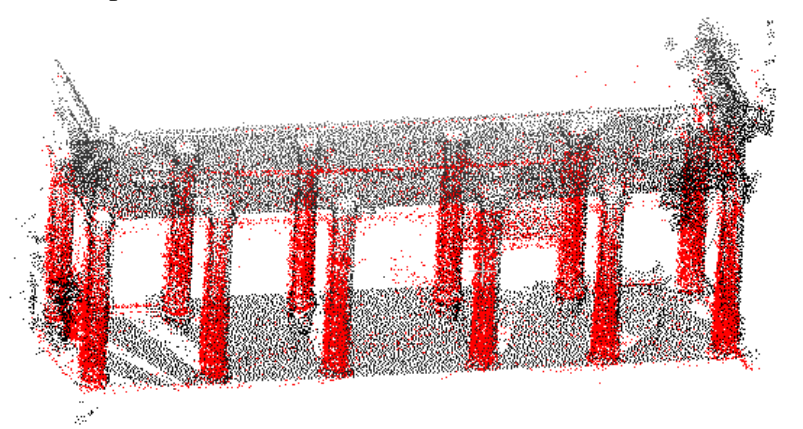

(b) Details (columns) collected by the two systems

Figure 8. Comparison result of robot system \& VZ400 point clouds in the outdoor scene (red represents point clouds collected by robot system, black represents point clouds collected by VZ400)

\section{CONCLUSIONS}

For the purpose of indoor and outdoor mapping, a low-cost wheeled robot-borne laser scanning system is proposed in this paper. The proposed robot laser scanning system is equipped with a laser scanner and an IMU. A globally consistent map can be achieved by the integration of 6-DOF pose estimation and the global optimization. Experiments show that the system has the ability to accomplish both indoor and outdoor mapping in a small area effectively. In the future, the research will focus on using the multi-sensor fusing LIDAR, camera and IMU to deal with more complex indoor scenes such as long corridor or tunnel environment.

\section{ACKNOWLEDGEMENTS}

The work presented in this article was substantially supported by National Natural Science Foundation Project of China (No. 41701530, 41725005 and 41531177), China Postdoctoral Science Found (No. 2018T110802), China Southern Power Grid Corporation Science and Technology Project (ZBKJXM20170229) and LIESMARS Special Research Funding and Key Laboratory of Spatial Data Mining \& 
Information Sharing of Ministry of Education, Fuzhou University (No. 2018LSDMIS06).

\section{REFERENCES}

Behley, J., Stachniss, C., 2018. Efficient surfel-based SLAM using 3D laser range data in urban environments, Robotics: Science and Systems (RSS).

Carlone, L., Kira, Z., Beall, C., Indelman, V., Dellaert, F., 2014. Eliminating conditionally independent sets in factor graphs: A unifying perspective based on smart factors, 2014 IEEE International Conference on Robotics and Automation (ICRA). IEEE, pp. 4290-4297.

Chen, C., Zou, X., Tian, M., Li, J., Wu, W., Song, Y., Dai, W., Yang, B., 2017. Low Cost Multi-Sensor Robot Laser Scanning System and its Accuracy Investigations for Indoor Mapping Application. The International Archives of Photogrammetry, Remote Sensing and Spatial Information Sciences 42, 83.

Deschaud, J.-E., 2018. IMLS-SLAM: scan-to-model matching based on 3D data, 2018 IEEE International Conference on Robotics and Automation (ICRA). IEEE, pp. 2480-2485.

Droeschel, D., Behnke, S., 2018. Efficient Continuous-time SLAM for 3D Lidar-based Online Mapping, 2018 IEEE International Conference on Robotics and Automation (ICRA). IEEE, pp. 1-9.

Grisetti, G., Stachniss, C., Burgard, W., 2007. Improved techniques for grid mapping with rao-blackwellized particle filters. IEEE transactions on Robotics 23, 34.

Huang, X., Cheng, X., Geng, Q., Cao, B., Zhou, D., Wang, P., Lin, Y., Yang, R., 2018. The apolloscape dataset for autonomous driving, Proceedings of the IEEE Conference on Computer Vision and Pattern Recognition Workshops, pp. 954-960.

Hunter, G., Cox, C., Kremer, J., 2006. Development of a commercial laser scanning mobile mapping systemStreetMapper, Proceedings of the 2nd International Workshop "The Future of Remote Sensing", Antwerp, Belgium, pp. 17-18.

Jaakkola, A., Hyyppä, J., Kukko, A., Yu, X., Kaartinen, H., Lehtomäki, M., Lin, Y., 2010. A low-cost multi-sensoral mobile mapping system and its feasibility for tree measurements. ISPRS journal of Photogrammetry and Remote Sensing 65, 514-522.

Li, J., Yang, B., Cong, Y., Cao, L., Fu, X., Dong, Z., 2019. 3D Forest Mapping Using A Low-Cost UAV Laser Scanning System: Investigation and Comparison. Remote Sensing 11, 717.

Liu, C., Wu, J., Furukawa, Y., 2018. Floornet: A unified framework for floorplan reconstruction from 3d scans, Proceedings of the European Conference on Computer Vision (ECCV), pp. 201-217.

Mao, Q., Zhang, L., Li, Q., Hu, Q., Yu, J., Feng, S., Ochieng, W., Gong, H., 2015. A least squares collocation method for accuracy improvement of mobile LiDAR systems. Remote sensing 7,7402-7424.

Neuhaus, F., Koß, T., Kohnen, R., Paulus, D., 2018. MC2SLAM: Real-Time Inertial Lidar Odometry Using Two-Scan Motion Compensation, German Conference on Pattern Recognition. Springer, pp. 60-72.

ROBOTIS, 2017. ROBOTIS Platform Turtlebot 3. URL: http://www.robotis.us/turtlebot-3/

Shan, T., Englot, B., 2018. LeGO-LOAM: Lightweight and Ground-Optimized Lidar Odometry and Mapping on Variable Terrain, 2018 IEEE/RSJ International Conference on Intelligent Robots and Systems (IROS). IEEE, pp. 4758-4765.

Smith, R., Self, M., Cheeseman, P., 1990. Estimating uncertain spatial relationships in robotics, Autonomous robot vehicles. Springer, pp. 167-193.

Wang, J., Sun, W., Shou, W., Wang, X., Wu, C., Chong, H.-Y., Liu, Y., Sun, C., 2015. Integrating BIM and LiDAR for real-time construction quality control. Journal of Intelligent \& Robotic Systems 79, 417-432.

Yu, F., Xiao, J., Funkhouser, T., 2015. Semantic alignment of LiDAR data at city scale, Proceedings of the IEEE Conference on Computer Vision and Pattern Recognition, pp. 1722-1731.

Zhang, J., Singh, S., 2014. LOAM: Lidar Odometry and Mapping in Real-time, Robotics: Science and Systems, p. 9. 